\title{
Inter-relation of Hypocalcaemia with Established Osteoporosis and DXA Analysis: A prospective study of 100 Indian subjects.
}

\author{
Bharat R. Dave ${ }^{1}$, Himanshu Kulkarni ${ }^{1}$, Devanand Degulmadi ${ }^{1}$, Shivanand Mayi ${ }^{1}$, \\ Ravi Ranjan Rai ${ }^{1}$, Kirit Jadav ${ }^{1}$, Ajay Krishnan ${ }^{1}$
}

\section{Abstract}

Purpose: The purpose of the study was to screen the presence of hypocalcemia and clinical signs specific to hypocalcemia in dualemission X-ray absorptiometry proven osteoporotic patients and also to analyze variations of $\mathrm{T}$ scores at specific anatomical regions in lumbar spine and hip.

Type: Prospective cohort.

Materials and Methods: One hundred patients who had T score of $<-2.5$ at any of the lumbar levels or in total lumbar T score were selected. Ionic calcium levels (normal - 1.1-1.135 mmol/L) of each patient were calculated. Trousseau's sign and Chvostek's sign were checked. Analysis of T scores was done for each patient.

Results: Twelve out of 100 patients had hypocalcemia. Out of whom, only one patient had positive Trousseau's sign and none had Chvostek's sign present. In normocalcemic patients $(n=88)$, seven patients had positive Trousseau's sign and three had Chvostek's sign present. Average total lumbar T score of 100 patients was $-3.0( \pm 1.1 \mathrm{SD})$. After calculating the averages, the L3 had least $\mathrm{T}$ score of $-3.3( \pm 0.9 \mathrm{SD})$ and L1 had highest $\mathrm{T}$ score of $-2.5( \pm 1.3 \mathrm{SD})$, respectively. Twenty-seven patients had total hip $\mathrm{T}$ scores $<-2.5$ and 72 patients had $T$ scores $<-2.5$ at Ward's triangle. Similarly, average total hip $T$ score of 100 patients was $-2.0( \pm 1.6 \mathrm{SD})$; average T score at Ward's angle was much lower at $-2.9( \pm 1.4 \mathrm{SD})$.

Conclusion: L3 vertebra and Ward's triangle are most sensitive indicators of osteoporosis. Although theoretically unlikely, hypocalcemia can be present in osteoporotic patients. Trousseau's sign and Chvostek's sign may be present in patients with established hypocalcemia; however, their absence does not rule out the diagnosis.

Keywords: Osteoporosis; Hypocalcemia; T score; Ward's triangle.

\section{Introduction}

Even after decades of research, osteoporosis still continues to gather attention of researchers all over the world. It has attained epidemic proportions because of increased life expectancy and other lifestyle factors [1]. Osteoporosis in 2001 was defined as "a skeletal disorder characterized by compromised bone strength, predisposing a person to an increased risk of fracture. Bone strength primarily reflects the integration of bone density and bone quality" [2]. The World Health Organization branded osteoporosis as a bone mineral density (BMD) $\mathrm{T}$ score $<-2.5$ as measured by dual-emission X-ray

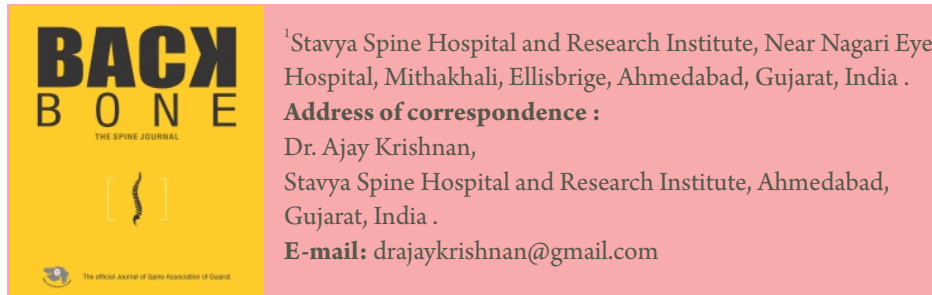

absorptiometry (DXA) [3]. DXA is considered to be a gold standard technique for the estimation of BMD due to its minimal radiation exposure, reproducibility, large normative data, and non-invasive nature [4]. Elucidation of DXA reports by a clinician is valuable as it dictates the further line of management. Interpretation of variety of $\mathrm{T}$ score patterns such as total hip score, total spine score, $\mathrm{T}$ scores of individual vertebrae, Ward's triangle, and trochanter can be confusing for diagnosis and management of osteoporosis. To add to the confusion, a practicing clinician comes across patients with established osteoporosis who have hypocalcemia in blood investigations and clinical signs such as Trousseau's sign and Chvostek's sign present. Even though it is an established fact that in calcium homeostasis, primary focus is on maintaining the serum ionic calcium levels, which in osteoporosis is achieved by parathyroid hormone-induced bone breakdown, very little literature is available that reports the coexistence of

(C) 2020 | Back Bone: The Spine Journal (The Official Journal Of "Spine Association Of Gujarat") | Available on www.backbonejournal.com | doi:10.13107/bbj.2020.v01i01.006 commercial use, distribution, and reproduction in any medium, provided the original work is properly cited. 


\begin{tabular}{|c|c|c|c|c|c|}
\hline \multicolumn{2}{|c|}{$\begin{array}{l}\text { Table 1: Clinical symptoms of } \\
\text { osteoporosis }\end{array}$} & \multicolumn{2}{|c|}{$\begin{array}{l}\text { Table 2: Number of cases with T- } \\
\text { score }<-2.5 \text { at each individual } \\
\text { vertebral level }\end{array}$} & \multicolumn{2}{|c|}{$\begin{array}{l}\text { Table 3: Average T score at } \\
\text { each individual vertebral level }\end{array}$} \\
\hline Symptoms & Number of & vertevial tever & Number of cases & Vertebral leve] & Average T-score \\
\hline Back nain & patients & vertebral level & with $\mathrm{T}$ score $<-2.5$ & L1 & $-2.5( \pm 1.3 \mathrm{SD})$ \\
\hline Rib pain & 98 & L1 & 55 & L2 & $-2.9( \pm 0.7 \mathrm{SD})$ \\
\hline Shin pain & 31 & $\begin{array}{l}\text { L2 } \\
\text { L3 }\end{array}$ & $\begin{array}{l}73 \\
82\end{array}$ & L3 & $-3.3( \pm 0.9 \mathrm{SD})$ \\
\hline Calf pain & 65 & L4 & 79 & L4 & $-3.1( \pm 1.0 \mathrm{SD})$ \\
\hline
\end{tabular}

osteoporosis and hypocalcemia [5]. In this study, the presence of hypocalcemia in patients who have been diagnosed with osteoporosis by DXA was explored and correlated with the presence of clinical signs specific to hypocalcemia. We also analyzed variations of $\mathrm{T}$ scores at specific anatomical regions in lumbar spine and hip to stress the importance of various anatomical considerations of T-score.

\section{Materials and Methods}

This was a prospective, observational study carried out at a single center. The study was not funded by any financial institution or company. The study was approved by the Institutional Ethical Committee and was registered with Clinical Trial Registry India (CTRI wide CTRI/2017/10/010195).

Inclusion criteria: Patients with age of more than 50 years, patients in whom DXA scan of hip and lumbar spine were performed and had T-score of $<-2.5$ at any of the lumbar levels, or in total lumbar T-score were selected till the planned cohort of 100 consecutive patients was completed. Patients on medical treatment for osteoporosis or hypocalcemia and those with a history of surgery in lumbar spine or with abnormal thyroid function tests or serum parathyroid tests were excluded from the study. Symptoms which were noted in every patient were in form of back pain, rib pain, shin pain, and calf pain (for osteoporosis). Trousseau's sign and Chvostek's sign (for hypocalcemia) were also checked in each of the patients. Ionic calcium levels (normal - 1.1-1.135 mmol/L) of each of the 100 selected patients were calculated by "Arsenzo III" method.

\section{Statistical analysis}

Patient demographics and characteristic categorical variables were analyzed, and the mean + standard deviation (minimummaximum) for all applicable variables were calculated.

\begin{tabular}{|c|c|c|}
\hline \multicolumn{3}{|c|}{$\begin{array}{l}\text { Table 4: Total number and average at of T-scores of } \\
\text { hip and Ward's triangle }\end{array}$} \\
\hline Type of T score & $\begin{array}{c}\text { Number of cases } \\
\text { with } \mathrm{T} \text { score }<-2.5\end{array}$ & $\begin{array}{l}\text { Average score (for } \\
100 \text { patients) }\end{array}$ \\
\hline Total hip score & 27 & $-2.0( \pm 1.6 \mathrm{SD})$ \\
\hline Ward's triangle & 72 & $-2.9( \pm 1.4 \mathrm{SD})$ \\
\hline
\end{tabular}

Results

Findings were analyzed in all 100 patients. Ninety-four patients in our study were female and six were male. Average age of the patients was 61.28 years (range, 50-86 years) years. Conventional symptoms correlating with osteoporosis were screened in all $10 \$ 0$ patients (Table 1). Shin pain was encountered the least and back pain was seen most commonly. After scanning the results of the ionic calcium levels, 12 (12\%) were noted to have hypocalcemia, with ionic calcium levels $<1.1 \mathrm{mmol} / \mathrm{L}$. Out of these 12 patients, only $1(8 \%)$ had positive Trousseau's sign and none had Chvostek's sign present. In the remaining 88 patients with normal calcium levels, 7 (8\%) patients had positive Trousseau's sign and 3 (3\%) had positive Chvostek's sign.

T-score was calculated at each vertebral level from L1 to L4 (Table 2). L3 was found to have maximum numbers of cases with T-score of -2.5 or below $(n=82)$ and L1 with least numbers of cases with T-score below-2.5 $(\mathrm{n}=55)$.

Twenty patients had total lumbar T-score more than -2.5 , however, it was $<-2.5$ at some vertebral level. Thirteen out of those were found at L3 vertebral level. Average total lumbar Tscore of 100 patients was found to be -3.0 ( $\pm 1.1 \mathrm{SD})$. After calculating the averages, $\mathrm{L} 3$ was one with least $\mathrm{T}$ score of -3.3 $( \pm 0.9 \mathrm{SD})$ and $\mathrm{L} 1$ had highest $\mathrm{T}$ score of $-2.5( \pm 1.3 \mathrm{SD}$ ) (Table $3)$.

T-scores of hip were also taken into consideration (Table 4). When total hip T-scores were taken into consideration, 27 patients had T-scores $<-2.5$. On the contrary, when T score of Ward's triangle was taken into consideration, 72 patients had $<-2.5$. Similarly, average total hip T-score of 100 patients was found to be $-2.0( \pm 1.6 \mathrm{SD})$. Average T-score at Ward's triangle was found to be at $-2.9( \pm 1.4 \mathrm{SD})$. Furthermore, out of 27 patients who had total hip T-score of $<-2.5,21$ patients had totallumbar T-score $<-2.5$.

\section{Discussion}

Calcium balance refers to the state of the body stores of calcium at equipoise over some extended time. The cumulative effect of intestinal absorption, renal, intestinal, and sweat gland excretion on bone calcium governs the calcium pool resulting in maintenance of calcium balance. The rate of bone 
absorption and resorption keeps on changing throughout the lifetime, and therefore, it does to the calcium balance. Children, in whom the formation is more than resorption, are in positive bone balance. Healthy young adults who have achieved peak bone mass have formation equal to resorption and are in neutral bone balance. However, in osteoporosis, a negative bone balance with resorption more than formation is characteristically noted, in elderly population [6]. Since the resorption of bone is mediated by parathyroid hormone that maintains serum calcium level; hypocalcemia may not be seen in patients with osteoporosis. However, no study has been conducted, as per our knowledge, to screen the incidence of hypocalcemia in osteoporosis till date [7]. While measuring the calcium levels, serum ionic (free) calcium levels are considered to be more sensitive than total calcium levels [8]. In our study, we measured serum ionic calcium levels to screen patients with hypocalcemia and found that 12 out of 100 patients had ionic calcium levels less than the lower threshold $(1.1 \mathrm{mmol} / \mathrm{L})$. This indicates although theoretically it is unlikely, hypocalcemia may present in osteoporotic patients.

The neuromuscular instability caused by hypocalcemia can be exhibited through the elicitation of Chvostek's sign and Trousseau's sign. Both of these signs are time honored physical predictors which are frequently associated with hypocalcemia [9]. Trousseau sign is elicited in hypocalcemia when the ionized calcium level is $1.75-2.25 \mathrm{mmol} / \mathrm{L}$. The hand adopts a characteristic posture when the sphygmomanometer cuff is inflated above the systolic blood pressure within $3 \mathrm{~min}$. The metacarpophalangeal joints are flexed, the interphalangeal joints of the fingers and thumb are extended, and the thumb adopts a posture of opposition [10]. Trousseau sign is more specific than Chvostek's sign for latent tetany, which can be caused by hypocalcemia, hypomagnesemia, and metabolic alkalosis.

In our study, we found that $7 \%$ of normocalcemic patients $(\mathrm{n}=88)$ had a positive Trousseau's sign. Although 12 is a small number of patients for a concrete conclusion, only 1 hypercalcemic patient out of $12(8 \%)$ had a positive Trousseau's sign and none had a positive Chvostek's sign. In their study, Schaaf et al. found that $94 \%$ of patients with confirmed hypocalcemia had a positive Trousseau's sign, whereas only $9 \%$ of normocalcemic demonstrated a positive Trousseau's sign [11]. This shows that Trousseau's sign and Chvostek's sign may be present in patients with established hypocalcemia but may as well be present in normocalcemic patients. However, mere absence of these signs does not rule out the diagnosis.

Patients with osteoporosis may present with a variety of symptoms. Most common symptom seen in our group of osteoporotic patients was back pain, which was present in 98 patients. Shin pain was the least encountered symptom, seen only in 31 patients. All these symptoms may or may not be attributed to osteoporosis since no symptoms have been established in literature which is specific to osteoporosis except for pain following a fracture [12].

Specificity of various $\mathrm{T}$ scores has been a debate for long in literature. Positioning, presence of previous fractures, presence of osteophytes, overlapping aortic calcific plaques, etc., can change the stipulated results at any vertebral level [13]. Furthermore, only referring to the total $\mathrm{T}$ scores at in lumbar spine can be deceptive and may give false-negative results [14]. Blake and Fogelman mentioned the possibility of precision error which may lead to suboptimal treatment by a clinician, labeling him without osteoporosis [15]. However, in our study, we observed that 20 patients had total T-score more than -2.5 that were labeled as osteopenic. In contrast, they had T-score $<-2.5$ in at least one vertebra from L1 to L4. Duboeuf et al. stated that $\mathrm{L} 3$ shows the most precise $\mathrm{T}$-scores when compared to other lumbar levels. For these various possibilities are postulated such as no overlapping of ribs for L1-2 or overlapping by iliac crest of L4 on lateral radiograph or L3 being the apex of lumbar lordosis, therefore being exactly parallel to X-ray beams etc. [16]. In our study, 82 patients had a T-score lower than -2.5 at L3 as compared to L1 (55 patients) that was the lowest. Similarly, average T score at L3 of all 100 patients was $-3.3( \pm 0.9 \mathrm{SD})$ as compared to $-2.5( \pm 1.3 \mathrm{SD})$ at L1. Furthermore, average total lumbar spine T score was $-3.0 \pm$ 1.1 SD. This indirectly shows that L3 is a better predictor of osteoporosis than total T-score and is the level that should be interpreted with caution by a clinician while interpreting the $\mathrm{T}$ scores of lumbar spine.

$\mathrm{T}$ scores of hip have also been scrutinized in the past. We observed that total hip T score was $<-2.5$ in only 27 patients. In a stark contrast to this finding, 72 patients had T-score $<-2.5$ when results from Ward's triangle were taken into consideration. There was a large difference between average scores of total hip and Ward's triangle. Average total hip T score was $-2.0( \pm 1.6 \mathrm{SD})$ and Ward's triangle $-2.9( \pm 1.4 \mathrm{SD})$. This finding shows how deceptive it would be to take the total hip score only into consideration for management. Yoshihashi et al. also stated that DXA of Ward's triangle is a sensitive indicator of osteoporosis and should be used to identify patients at increased risk for osteoporosis-related fractures and their management [17]. Out of 27 patients who had total hip Tscore $<-2.5,21$ had total lumbar T-score $<-2.5$. This indicates that there was 59 patients who had total $\mathrm{T}$ score $<-2.5$ at lumbar spine, not at hip. Hence, spine may be an accurate indicator of osteoporosis than hip when total scores are taken into consideration. Similar results were found by Liu et al. and Ma et al., in their respective studies $[18,19]$.

As per our knowledge, this is the first study that has screened the presence of hypocalcemia in osteoporotic patients. The 
limitation of our study is that we did not take other causes of hypocalcemia such as acute and chronic renal failure, Vitamin $\mathrm{D}$ deficiency, hypoalbuminemia, magnesium deficiency, and pancreatitis into consideration while screening the patients for our study. Furthermore, $\mathrm{Z}$ score interpretation has not been taken into consideration in our study. However, we feel that our initial results are encouraging and will focus more information in future studies.

\section{Conclusion}

L3 vertebra and Ward's triangle are most sensitive indicators of osteoporosis when DXA of hip and lumbar spine is carried out and analysis should not be done looking only at total hip or lumbar spine scores which could be illusive. Average T score should not be used as a management guide; instead, we should be using the least value to guide the treatment protocol. Although theoretically unlikely, hypocalcemia can be present in osteoporotic patients. Trousseau's sign and Chvostek's sign may be present in patients with established hypocalcemia or normocalcemic. However, its absence does not rule out the diagnosis.

\section{Summary}

We screened presence of hypocalcemia its clinical signs in osteoporotic patients and analyzed variations of $\mathrm{T}$ scores. We observed that L3 and Ward's triangle are most sensitive indicators of osteoporosis. Hypocalcemia can be present in osteoporotic patients. Trousseau's sign and Chvostek's sign may or may not be present in hypocalcemic patients.

\section{References}

1. Mithal A, Dhingra V, Lau E, Stenmark J, Nauroy L. The Asian Audit: Epidemiology, Costs and Burden of Osteoporosis in Asia. Nyon, Swizterland: International Osteoporosis Foundation; 2009.

2. Osteoporosis prevention, diagnosis, and therapy. NIH Consens Statement 2000;17:1-45.

3. Kanis JA. Assessment of fracture risk and its application to screening for postmenopausal osteoporosis: Synopsis of a WHO report. WHO study group Osteoporos Int 1994;4:368-81.

4. Blake GM, Fogelman I. The role of DXA bone density scans in the diagnosis and treatment of osteoporosis. PostgradMed J2007;83:509-17.

5. Boden SD, Kaplan FS. Calcium homeostasis. Orthop Clin North Am 1990;21:3142.

6. Peacock M. Calcium metabolism in health and disease. Clin J Am Soc Nephrol 2010;5:S23-30

7. Li Z, Kong K, Qi W. Osteoclast and its roles in calcium metabolism and bone development and remodeling. Biochem Biophys Res Commun 2006;343:34550 .

8. Sava L, Pillai S, More U, Sontakke A. Serum calcium measurement: Total versus free (ionized) calcium. Indian J Clin Biochem 2005;20:158-61.

9. Urbano FL. Signs of hypocalcemia: Chvostek's and Trousseau's signs. Hosp Physician 2000;36:43-5.

10. Shoback D, Marcus R, Bikle D. Metabolic bone disease. In: Greenspan FS, Gardner DG, editors. Basic and Clinical Endocrinology. 3rd ed. Los Altos, CA: Lange Medical Publications; 2004.p. 324.

Conflict of Interest: NIL Source of Support: NIL
11. Schaaf M, Payne CA. Effect of diphenylhydantoin and phenobarbital on overt and latent tetany. NEnglJMed 1966;274:1228-33.

12. Watts NB. Postmenopausal osteoporosis: A clinical review. J Womens Health (Larchmt) 2018;27:1093-6.

13. Garg MK, Kharb S. Dual energy X-ray absorptiometry: Pitfalls in measurement and interpretation of bone mineral density. Indian J Endocrinol Metab 2013;17:203-10.

14. Ramos RL, Armán JA, Galeano NA, Hernández AM, Gómez JG, Molinero JG. Absorciometría con rayos $X$ de doble energía. Fundamentos, metodologia $y$ aplicaciones clínicas. Radiologia 2012;54:410-23.

15. Blake GM, Fogelman I. An update on dual-energy x-ray absorptiometry. Semin NuclMed 2010;40:62-73.

16. Duboeuf F, Pommet R, Meunier PJ, Delmas PD. Dual-energy X-ray absorptiometry of the spine in anteroposterior and lateral projections. Osteoporos Int 1994;4:110-6.

17. Yoshihashi AK, Drake AJ, Shakir KM. Ward's triangle bone mineral density determined by dual-energy $x$-ray absorptiometry is a sensitive indicator of osteoporosis. Endocr Pract 1998;4:69-72.

18. Liu X, Qian ZY, Feng YS, Li HL, Xu YJ. Comparison of differences between hip and lumbar bone mineral density in dual energy X-ray absorptiometric data. Zhonghua YiXueZaZhi2013;93:191-4.

19. Ma XH, Zhang W, Wang Y, Xue P, Li YK. Comparison of the spine and hip BMD assessments derived from quantitative computed tomography. Int J Endocrinol 2015;2015:675340.

\section{How to Cite this Article}

Dave BR, Kulkarni H, Degulmadi D, Mayi S, Rai RR, Jadav K, Krishnan A| Inter-relation of Hypocalcemia with Established Osteoporosis and DXA Analysis - A Prospective Study of 100 Indian Subjects | Back Bone: The Spine Journal | October 2020-March 2021; 1(1): 19-22. 